\title{
Bow shock specularly reflected ions in the presence of low-frequency electromagnetic waves: a case study
}

\author{
K. Meziane' ${ }^{1}$, C. Mazelle ${ }^{2}$, M. Wilber ${ }^{3}$, D. LeQuéau ${ }^{2}$, J. P. Eastwood ${ }^{4}$, H. Rème ${ }^{2}$, I. Dandouras ${ }^{2}$, J. A. Sauvaud ${ }^{2}$, \\ J. M. Bosqued ${ }^{2}$, G. K. Parks ${ }^{3}$, L. M. Kistler ${ }^{5}$, M. McCarthy ${ }^{6}$, B. Klecker ${ }^{7}$, A. Korth ${ }^{8}$, M.-B. Bavassano-Cattaneo ${ }^{9}$, \\ R. Lundin ${ }^{10}$, and A. Balogh ${ }^{4}$ \\ ${ }^{1}$ Physics Department, University of New Brunswick, Canada \\ ${ }^{2}$ Centre d'Etudes Spatiales des Rayonnements, 9 Avenue du Colonel Roche, Toulouse, 31028, France \\ ${ }^{3}$ Space Sciences Laboratory, University of California, Berkeley 94720, USA \\ ${ }^{4}$ Space and Atmospheric Physics, The Blackett Laboratory, Imperial College, London, SW7 2BW, UK \\ ${ }^{5}$ Space Science Center, University of New Hamshire, Durham, 03824, USA \\ ${ }^{6}$ Earth and Space Sciences, University of Washington, Seattle, 98195, USA \\ ${ }^{7}$ MPI für Extraterrestrische Physik, Garching, 85741, Germany \\ ${ }^{8}$ MPI für Aeronomie, Katlenburg-Lindau, 37191, Germany \\ ${ }^{9}$ CNR-IFSI, Roma, 00133, Italy \\ ${ }^{10}$ Swedish Institute of Space Physics, Kiruna, 98128, Sweden
}

Received: 9 October 2003 - Revised: 9 February 2004 - Accepted: 25 February 2004 - Published: 14 July 2004

Part of Special Issue "Spatio-temporal analysis and multipoint measurements in space"

\begin{abstract}
An energetic ion ( $E \leq 40 \mathrm{keV})$ event observed by the CLUSTER/CIS experiment upstream of the Earth's bow shock is studied in detail. The ion event is observed in association with quasi-monochromatic ULF MHD-like waves, which we show modulate the ion fluxes. According to three statistical bow shock position models, the Cluster spacecrafts are located at $\sim 0.5 R_{E}$ from the shock and the averaged bow shock $\theta_{B \mathrm{n} 0}$ is about $\sim 30^{\circ}$. The analysis of the threedimensional angular distribution indicates that ions propagating roughly along the magnetic field direction are observed at the onset of the event. Later on, the angular distribution is gyrophase-bunched and the pitch-angle distribution is peaked at $\alpha_{0} \sim \theta_{B \mathrm{n} 0}$, consistent with the specular reflection production mechanism. The analysis of the waves shows that they are left-handed in the spacecraft frame of reference (right-handed in the solar wind frame) and propagate roughly along the ambient magnetic field; we have found that they are in cyclotron-resonance with the field-aligned beam observed just upstream. Using properties of the waves and particles, we explain the observed particle flux-modulation in the context of $\theta_{B \mathrm{n}}$ changes at the shock caused by the convected ULF waves. We have found that the high count rates coincide with particles leaving the shock when $\theta_{B \mathrm{n}}$ angles are less than $\sim 40^{\circ}$, consistent with the specular reflection hypothesis as the production mechanism of ions.
\end{abstract}

Correspondence to: $\mathrm{K}$. Meziane

(meziane@unb.ca)

\section{Introduction}

Several types of ion populations have been observed to emerge from the Earth's bow shock into the upstream regions. For an in-depth discussion of these, readers should consult reviews by Fuselier (1994). Here, we are concerned only with field-aligned beams (FABs) and gyrating ions, and will therefore limit our discussion to these. An important parameter strongly linked to the types of upstream ions observed is the angle $\theta_{B \mathrm{n}}$ between the interplanetary magnetic field (IMF) direction and the local bow shock normal. In quasi-perpendicular regions with $40^{\circ} \lesssim \theta_{B \mathrm{n}} \lesssim 70^{\circ}$, we typically observe FABs, which are ions of a few to $\sim 15 \mathrm{keV}$ that propagate away from the bow shock collimated along the magnetic field at 1 to several times the solar wind speed (Bonifazi and Moreno, 1981a). Downstream of the fieldaligned beam region, ion populations that are characterized by peaks in phase space density (PSD) at non-zero pitchangles are often seen. When these distributions are gyrotropic they form rings in velocity space; otherwise, they gyrate around the magnetic field as a bunch. Gyrating ions are often observed in association with ultra-low-frequency (ULF) waves having substantial amplitude (Fuselier et al., 1986a). The waves are right-handed and propagate nearly along the ambiant magnetic field (Meziane et al., 2001). It is believed that the ULF waves are excited through a beam plasma instability resulting from the propagation of fieldaligned ions which preceed them closer to the foreshock boundary (Gary et al., 1981). 
CIS-HIA RUMBA (SC 1) 09/Mar/2002

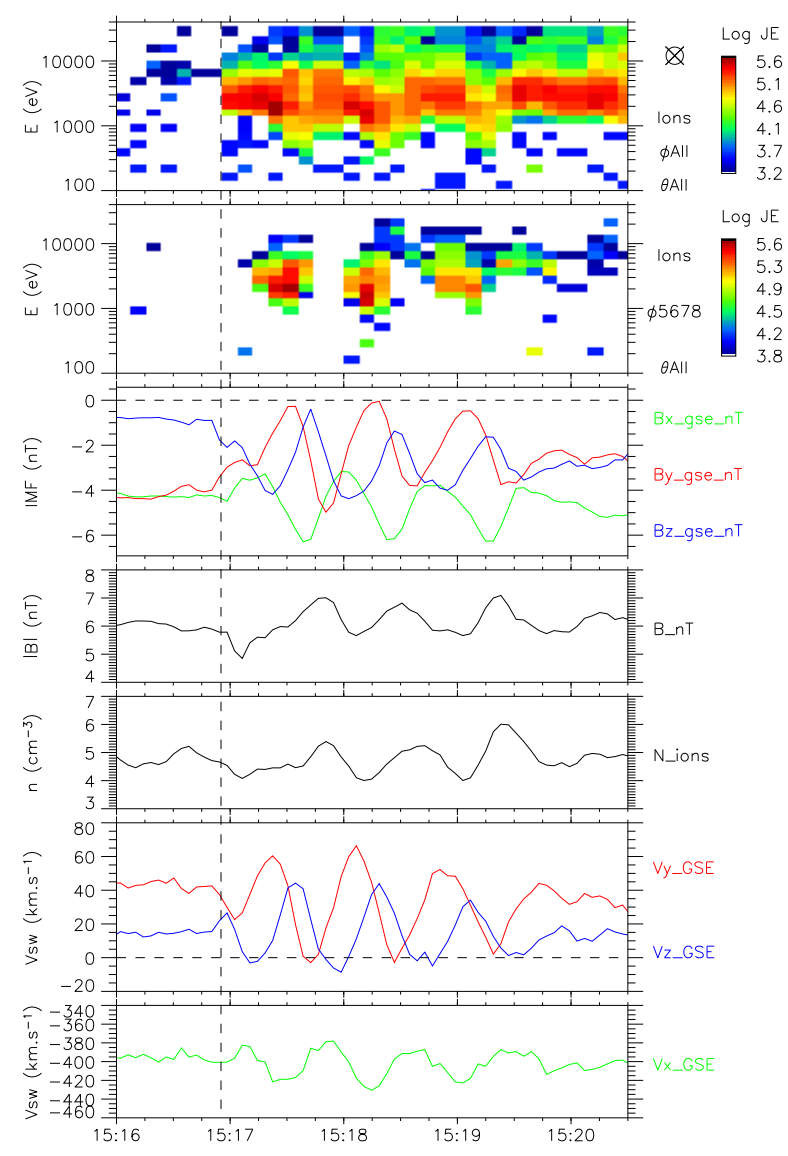

Fig. 1. The top two panels show, respectively, energy spectrograms from HIA-G integrated over all directions, and over the $145-235^{\circ}$ azimuthal look directions; the third and fourth panels display the GSE components and magnitude of the IMF; and the last three panels present solar wind densities and GSE velocities from HIA-g. The dashed vertical line indicates the magnetic connection time.

Two mechanisms are put forward to explain the origin of gyrating ions. In one mechanism, the waves produced through a beam-plasma instability can, in turn, trap the ions and cause the phase-bunching of the distribution (Hoshino and Terasawa, 1985). Since the field-aligned beams are known to propagate to distances far in excess of $100 R_{E}$ from the shock, the local production of gyrating ions through this process may be observed very far from the shock (Meziane et al., 2001). In the second mechanism, a portion of the incoming solar wind is reflected in a specular manner at the shock (Gosling et al., 1982; Gurgiolo et al., 1983). When propagating upstream, the bunched ions undergo gyrophase mixing within a few Earth radii from their source on the shock, leading to nearly gyrotropic distribution functions (Gurgiolo et al., 1983). Two-dimensional measurements suggest the presence of gyrating gyrotropic (ring) distributions within $\sim 4 R_{E}$ of the bow shock (Fuselier et al., 1986a). However, inspection of fully 3-D observations have consistently shown the presence of distributions that are nongyrotropic.
To date, only a few studies have focused specifically on the specular reflection process. Gosling et al. (1982) reported the first evidence of specular reflection of a portion of the solar wind using the ISEE-FPE experiment, at a time when ISEE was just upstream of the shock. Another event consistent with specular reflection observed at $\sim 4 R_{E}$, and in association with ULF waves, was studied by Thomsen et al. (1985).

At the present time, we ignore how the shock geometry affects the specular reflection mechanism efficiency. However, as shown by Gosling et al. (1982) only particles reflected for $\theta_{B \mathrm{n}}<45^{\circ}$ (quasi-parallel geometry) have guiding centers oriented upstream. However, analysis of orbital motions shows that not all of these will escape the shock, as gyromotions will carry particles back across the shock for $\theta_{B \mathrm{n}}$ values as low as $39.9^{\circ}$ (Schwartz et al., 1983).

In the presence of large amplitude ULF waves near the shock, which are always observed in association with gyrating ions, the local bow shock $\theta_{B \mathrm{n}}$ varies substantially. Large $\Delta B / B$ perturbations of the magnetic field substantially affect the local geometry where they intersect the shock. A result is that the shock geometries may switch back and forth from quasi-parallel to quasi-perpendicular (Greenstadt and Mellott, 1985). Using a simple geometrical model, Fuselier et al. (1986b) have shown that this effect, depending upon the wave characteristics, should inhibit the escape of specularly reflected ions. Straightforward geometric arguments indicate that when the wave amplitude is high $(\Delta B / B \sim 1)$, the effect is relatively strong. However, there has not yet been any observational substantiation of this effect in the presence of large amplitude magnetohydrodynamic (MHD) waves.

Here, we report on Cluster/CIS observations that are consistent with specularly reflected ions in the presence of monochromatic ULF waves. In Sect. 2 we briefly describe the Cluster/CIS experiment. The observations and a wave analysis are presented in Sect. 3, followed by the determination of the shock geometry in Sect. 4 . We then compare the observations with properties expected for the specular reflection mechanism in Sect. 5. In Sect. 6 we demonstrate that the intermittently observed gyrating particle distributions are consistent with changing local shock geometries modulated by the large-amplitude waves. Finally, we present a summary of observations and conclusions in Sect. 7.

\section{Experiment}

The particle data used in this study are from the Cluster Ion Spectrometer experiment, which includes i) a Hot Ion Analyser (HIA) that measures particles in the energy range $0.005-$ $32 \mathrm{keV}$; ii) a mass spectrometer (CODIF), which combines a top-hat electrostatic analyzer with a time-of-flight section to measure the major species $\mathrm{H}^{+}, \mathrm{He}^{+}, \mathrm{He}^{++}$and $\mathrm{O}^{+}$over the energy range $0.02-38 \mathrm{keV} / \mathrm{q}$. Both instruments measure full 3 -D distributions within one satellite spin period $(4 \mathrm{~s})$ with an angular resolution of $22.5^{\circ} \times 22.5^{\circ}$. In normal telemetry mode, one distribution is transmitted every 2 or 3 spins, while in burst mode a distribution is provided each spin. 
Both analyzers have high and low geometry factor sections. The low geometry factor all-ion analyzer (HIA-g) records solar wind beam and magnetosheath fluxes without saturation, while the high geometry factor analyzer (HIA-G) is wellsuited for measuring the orders of magnitude lower fluxes of upstream ions. Extensive descriptions of this instrument are provided in Rème et al. (2001). Our study also uses magnetic field data, which are provided by the on-board fluxgate magnetometer (FGM) (Balogh et al., 2001). We have used 4-s-averaged field components to investigate the association of low-frequency waves with the backstreaming ions. The Cluster polar orbits have apogees within several $R_{E}$ of the average shock location, and as a result multiple crossings are common, with most observations of upstream particles occuring in proximity to this boundary. Because of the very small $(145 \mathrm{~km})$ interspacecraft separation, the particle instrument observations were nearly identical, and we have chosen to use those from SC 3.

\section{Observations and data analysis}

\subsection{Particle data}

Figure 1 shows observations from 9 March 2002 from 15:1615:22 UT, while Cluster SC 1 was at $(\mathrm{X}=+15.70, \mathrm{Y}=+0.31$, $\mathrm{Z}=+5.985) R_{E}$ (GSE). The top two panels show the energy spectrograms measured by the CIS/HIA high-geometry factor sensor. The top panel was constructed from integrating fluxes recorded in all directions, whereas the second included only those counts within the sector defined by $145-235^{\circ}$ azimuthal look direction (i.e. those particles moving dominantly sunward). The third and fourth panels show, respectively, the magnetic field components and magnitude from FGM. Finally, the last three panels show the solar wind ion densities and velocities from the low-geometry factor HIAg sensor. Between 15:16:48 and 15:16:56 UT, Cluster SC 1 becomes connected to the bow shock due to an IMF rotation. Three "spots" of enhanced ion fluxes are revealed on the second spectrogram show (backstreaming) ions with guiding center velocities oriented mainly sunward. They appear to be coincident with maxima of the $B_{y}$ component of the nearly monochromatic ULF waves observed by FGM until 15:19 UT.

Figure 2 displays 8-s (2-spin) snapshots of distributions measured at successive times spanning 80-s following the shock connection time indicated in Fig. 1, for $E \sim 6 \mathrm{keV}$ and $E \sim 3.3 \mathrm{keV}$. The Hammer-Aitoff projection is used to display the full $4 \pi$ sr solid angle coverage of HIA-G for the two energy channels (Meziane et al., 2001; Mailing, 1992), and in combination present a portion of the full 3-D distributions measured by CIS/CODIF. For these contours of constant pitch-angle less than $90^{\circ}$ appear as ovals, and curves of constant gyrophase radiate from the center. Therefore, each frame in Fig. 2 is aproximately a polar plot of PSDs in gyrophase and pitch-angle for a single energy in the solar wind frame of reference. The diamond at the center represents the anti-field-aligned direction, which points out of the page, and the solar wind direction is indicated by the asterisk. The dashed lines appearing in each panel trace out lines of constant GSE polar and azimuthal angle. These energies were chosen because the ion energy spectrum, sampled in the solar wind frame of reference, peaked at $E \sim 5-6 \mathrm{keV}$ for the first snapshot and at $E \sim 2.5-3.5 \mathrm{keV}$ in the later ones.

The first snapshot taken at 15:16:48-56 UT indicates an ion beam propagating along $\mathbf{- B}$ direction with parallel speed $V_{B}=1060 \pm 100 \mathrm{~km} \mathrm{~s}^{-1}$. Due to a rapid IMF rotation, the cone angle varies from a value of $47^{\circ}$ at 15:16:48-15:16:52 to $43^{\circ}$ at 15:17:00-15:19:44 UT, placing Cluster SC 1 within a region of gyrating ions, as indicated by the 3-D plots starting from 16:17:00 UT. Gyrating ions are identified by their gyrophase-restricted distribution peaked off the magnetic field direction. When constructing the distributions for Fig. 2, we averaged the IMF over the time interval 15:17:00 15:19:44 UT - an integral number of wave periods. Consequently, their instantaneous velocities are modulated by the wave, but the parallel and perpendicular velocities thus obtained are with respect to the time average orientation of the field and bow shock normal.

We also mention that during the same time interval, the cyclotron period is about $\sim 11 \mathrm{~s}-$ slightly larger than the snapshot integration time $(8 \mathrm{~s})$, however, two subsequent snapshots are separated by $12 \mathrm{~s}$. We have conducted a quantitative analysis on the gyrating distributions above and have found that they are peaked at a pitch-angle $\alpha_{0}=30^{\circ}$.

\subsection{Wave analysis}

Prominent large amplitude low-frequency waves displayed in the third panel of Fig. 1 on the DC magnetic field components appear after 15:17:12 UT, the very time when the energetic backstreaming ion distributions in Fig. 2 change from fieldaligned beams to a gyrating form.

The observed period in the spacecraft frame, computed from the mean interval between magnetic field wave crests for all components, is $T_{\mathrm{obs}}=46.6 \pm 2.0 \mathrm{~s}$, while the local proton gyroperiod $T_{\text {cyl }}$ is about $11 \mathrm{~s}$.

We have applied the standard minimum variance analysis (MVA) technique (see, e.g. Sonnerup and Scheible, 1998, and references therein) to characterize the waves, and have determined their polarization and direction of propagation with respect to the background magnetic field $\mathbf{B}_{0}$. The usual convention is used to order the eigenvalues of the covariance matrix of the field perturbations $\lambda_{1}>\lambda_{2}>\lambda_{3}$ (maximum, intermediate and minimum variances, respectively). The direction of minimum variance gives the direction of propagation with respect to $\mathbf{B}_{0}$ computed as the averaged field vector during the time interval analysed, and provides the angle $\theta_{k B}$ between the wave vector $\mathbf{k}$ and $\pm \mathbf{B}_{0}$. The quality of this determination is measured via the value of the eigenvalue ratio. The angular error on the wave propagation direction is estimated by $\Delta \theta_{k B}=\sqrt{\left(\frac{\lambda_{3}}{(M-1)} \frac{\lambda_{2}}{\left(\lambda_{2}-\lambda_{3}\right)^{2}}\right)}, M$ being the number of vectors. The polarization of the field perturbation can then be 


\section{CLUSTER CODIF-1 Product 13 2002-03-09}
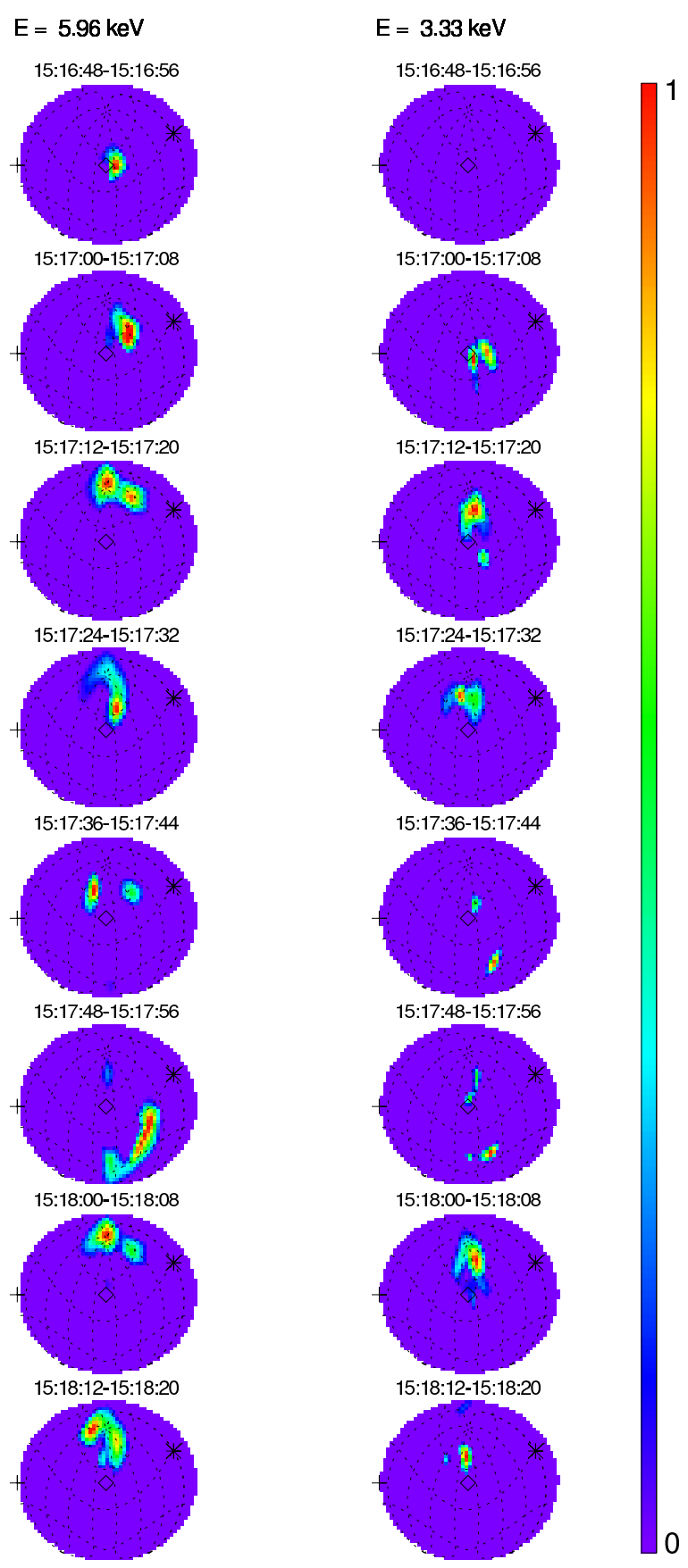

Fig. 2. Energy slices from 8 -s $3-\mathrm{D}$ measurements of ion distributions from CIS/CODIF. The $\sim 6 \mathrm{keV}$ and $\sim 3.4 \mathrm{keVpanels}$ each show $4 \pi$ sr of solid angle for a detector energy channel. The diamond at the center represents the anti-field aligned direction, which is directed out of the page, and the solar wind direction is indicated by the "*" symbol. Each separately normalized panel represents phase space densities in the solar wind frame of reference.

determined with respect to the ambient field $\mathbf{B}_{0}$ in the spacecraft frame. Next, we computed the fluctuation levels of the magnetic field and the solar wind proton parameter perturbations, i.e., the magnetic field compression ratio $\delta|\mathbf{B}| / B_{0}$, the ion density compression ratio $\delta N_{i} / N_{i}$, and the ratio $|\delta \mathbf{B}| / B_{0}$ that characterizes the amplitude of the wave field.
Figure 3 illustrates the results obtained for satellite 1, for the time interval 15:17:14-15:19:38 UT. The upper left panel shows the time sequences of the magnetic field components in the principal axes coordinate system, and the other panels present the corresponding hodograms. Despite the large amplitude of the waves, the waveform is regular 
and quasi-monochromatic, and the hodograms reveal that the wave field vector remains in a well-defined plane. The normal vector $\left(\lambda_{2} / \lambda_{3}=46.0\right)$ was determined to high accuracy, with $\Delta \theta_{k B} \approx 1^{\circ}$. The minimum variance direction is $\mathbf{e}_{\min }=(0.65,0.18,0.74)$ in GSE coordinates. This provides a direction of propagation nearly parallel to the ambient magnetic field $\mathbf{B}_{0}$, computed as the averaged field for the time interval studied $\left(\theta_{k B}=15^{\circ} \pm 1\right)$. The waves are nearly circularly $\left(\lambda_{1} / \lambda_{2}=1.1\right)$ and left-hand polarized with respect to $\mathbf{B}_{0}$ in the spacecraft frame, as shown on the hodogram in the principal variance plane, with an observed period (Doppler-shifted) much larger than the local proton gyroperiod. As expected, the computed total wave amplitude is very large $\left(|\delta \mathbf{B}| / B_{0} \approx 0.55\right)$. The wave is mainly transverse $\left(\left|\delta B_{\perp}\right| / B_{0}=0.48\right)$ but the compressive component is not negligible, with $\left|\delta B_{\|}\right| / B_{0}=0.22$ comparable to the relative ion density variation $\delta N_{i} / N_{i} \sim 0.2$. The low-frequency waves observed in the IMF on the three other satellites are very similar because of the small satellite separation, leading to nearly-identical MVA results (Mazelle et al., 2003). The angle between the four spacecraft averaged magnetic field and the averaged MVA direction $\left(\mathbf{e}_{\mathrm{mva}}=(0.71,0.21,0.67) \pm 0.01\right.$ in each component) is $15.1^{\circ}$. The multi-spacecraft timing analysis techniques (e.g. Eastwood et al., 2002, 2003) do not give reliable results for the plasma frequency and phase velocity because of the small separation $(\sim 100 \mathrm{~km})$ between the four spacecraft, which leads to a very small time separation between wave fronts. However, it is helpful to confirm that the waves propagate upstream. As we will detail later, it is possible to derive a first estimate of the wavelength by taking into account the Doppler shift between the plasma and spacecraft frames, and by assuming that the wave frequency in the plasma frame is much smaller than the observed frequency (ULF wave hypothesis). This gives a value $\lambda_{\text {exp }} \sim 12000 \mathrm{~km}$ that is many times the spacecraft separation scale, and accounts for the very similar observations. Note also that these foreshock ULF waves have properties typical of previous observations (Fuselier et al., 1986a). They are mainly transverse, propagate close to the background field, with a phase velocity close to the Alfvèn speed and co-streaming with the foreshock backstreaming particles, i.e. their propagation is mainly against the solar wind flow, so that they are subject to anomalous Doppler shift and polarization reversal when viewed from the plasma frame. They turn out to be right-hand mode waves, with a very low-frequency in the plasma frame that is typically $\Omega_{p} / 10$, and have a wavelength of the order of $1-2 R_{E}$. The properties of the observed waves are summarized in Table 1.

\section{Shock geometry}

Before looking at the wave effects on the particle dynamics, an estimation of the angle $\theta_{B \mathrm{n}}$ as well as the distance from the shock are needed. For this purpose a bow shock model is adopted. The location of the Earth's bow shock has been the subject of several observational and theoretical investi-
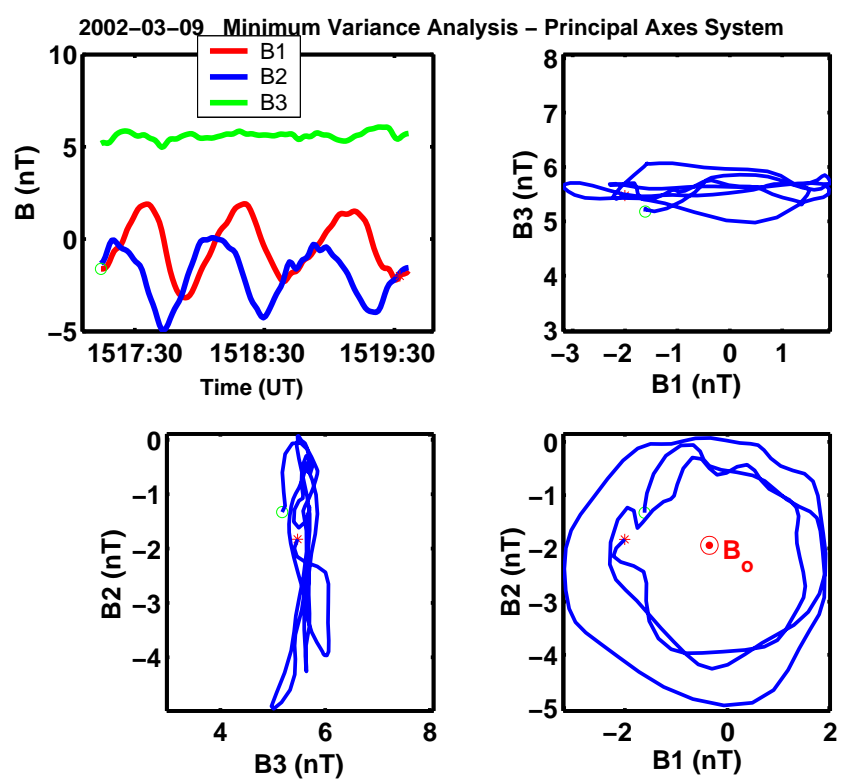

Fig. 3. Principle axis components (upper left) and corresponding hodograms of the low-frequency wave magnetic field in the principal axes coordinates derived from a minimum variance analysis for the event shown in Fig. 1. Open circles (asterisks) indicate the beginning (end) of each hodogram.

gations. An early study by Fairfield (1971) assumed a 2-D shock and ignored the effects of solar wind dynamic pressure. Formisano (1979) was the first to use a 3-D bow wave shape, including the effect of solar wind dynamic pressure. Slavin and Holzer (1981) conducted an extensive study using data from multiple spacecraft, including those used by Fairfield (1971) and Formisano (1979). They found that the Earth's bow shock shape is well represented by an eccentric conic when taking into account the variations of the solar wind dynamical pressure. A more extensive study using data from 17 different spacecraft was reported by Peredo et al. (1995). This uses a 3-D bow shock shape, and includes the effects of solar wind variations parameterized by the sonic $M_{S}$, Alfvènic $M_{A}$ and magnetosonic $M_{M S}$ Mach Numbers, as well as the orientation of the IMF direction. According to this study, $M_{A}$ controls the shape of the shock more strongly than $M_{S}$ and $M_{M S}$. Another model by Cairns et al. (1995) emphasizes the MHD effects at low Mach numbers. Finally, a gas dynamics-based model using 351 independent bow shock crossings by the ISEE-1 spacecraft has been reported by Farris et al. (1991). They found that these data fit a hyperboloid shape well. For comparison purposes, we use the Farris et al. (1991), Cairns et al. (1995), and Slavin and Holzer (1981) models. We do not use the recent Peredo et al. (1995) model because it has been shown to overestimate the bow shock stand-off position by as much as $20 \%$ (Merka et al., 2003).

Table 2 lists the distance from the bow shock along the magnetic field direction and $\theta_{B \mathrm{n} 0}$ as obtained from the three bow shock models mentioned above. The aberrated-GSE coordinates $\left(x_{S}, y_{S}, z_{S}\right)$, where the IMF field line threading 
Table 1. Properties of ULF waves associated with the gyrating ion distributions.

\begin{tabular}{lcccccccccc}
\hline Time interval & $\lambda_{2} / \lambda_{3}$ & $\lambda_{1} / \lambda_{2}$ & $\theta_{k B}, \operatorname{deg}$ & $\theta_{k V}, \operatorname{deg}$ & $|\delta \mathbf{B}| / \mathrm{B}$ & $\delta|\mathbf{B}| / \mathrm{B}$ & $T_{\text {obs }}, s$ & $T_{\text {cyl }}, s$ & $T_{\text {pred }}, s$ & $\lambda_{\|}, R_{E}$ \\
\hline 09 March 2002 & & & & & & & & & & \\
15:17:14-19:38 UT & 46.0 & 1.1 & $15 \pm 1$ & $130 \pm 2$ & .55 & .22 & $46.6 \pm 2$ & 11 & 46.5 & 1.9 \\
\hline
\end{tabular}

Table 2. Intersection of the IMF line with the bow shock.

\begin{tabular}{lcccccc}
\hline Bow shock model & $D_{S}, R_{E}$ & $\theta_{B \mathrm{n} 0}, \mathrm{deg}$ & $\theta_{V \mathrm{n}}, \mathrm{deg}$ & $x_{S}, R_{E}$ & $y_{S}, R_{E}$ & $z_{S}, R_{E}$ \\
\hline Farris et al. (1991) & .43 & 28 & 161 & 16.0 & 1.6 & 6.2 \\
Cairns et al. (1995) & .48 & 31 & 164 & 15.3 & 1.2 & 5.7 \\
Slavin and Holzer (1981) & .39 & 29 & 162 & 15.9 & 1.6 & 6.2 \\
\hline
\end{tabular}

the spacecraft intersects the bow shock, are also indicated. Average values of the magnetic field direction and solar wind plasma moments for the time of interest, 15:16:0015:20:00 UT, have been used for the determination of the quantities listed on Table 2. The three bow shock models provide similar values for the distance $D_{S}$ and the angles $\theta_{B \mathrm{n} 0}$ and $\theta_{V \mathrm{n}}$. The distance shown here is less than the wavelength for the associated waves, computed in Sect. 6 below. However, it is higher than the $\sim 0.2 R_{E}$ gyroradii of the observed particles, indicating that these are not re-encountering the shock in their subsequent motion. Finally, we note that the models agree on the location of $S$ to within $1 R_{E}$.

\section{The specular reflection mechanism}

In this section we examine specular reflection as a possible mechanism for the production of gyrophase-bunched distributions. Gosling et al. (1982) first reported few-keV bow shock-reflected ions at quasi-parallel shock that could be explained kinematically by a specular reflection process, whereby solar wind ions returning from the shock initially have their normal velocities reversed with their tangential velocities unchanged, as seen in the de Hoffman-Teller frame. The physical mechanism underlying this probably involves a combination of magnetic and electrostatic forces present at the shock layer (Gosling and Robson, 1985). Specularly reflected particles emerge from the shock at specific pitchangles and gyrophases, and therefore should lead to nongyrotropic gyrating distributions, but we are not aware of thorough treatments of this in the literature.

In this section we show that the kinematics of specular reflection of solar wind ions leads to gyrophase-restricted upstream distributions. First, we assume a locally planar, infinitesimally thin shock in the yz-plane. We only consider shock encounters by thermal (solar wind) ions for which the typical ion gyroradius is on the order of the shock thickness. It is likely that incoming ions undergo many reflections before they eventually escape upstream, but we treat the whole process as equivalent to a single reflection that reverses the normal component. In view of this, we make no assumptions about an infinitesimally thin shock layer. Without loss of generality, we orient our $\mathrm{x}$-axis along the local shock normal n. Near the shock surface we assume an uniform upstream magnetic field $\mathbf{B}_{0}$ that makes an angle $\theta_{B \mathrm{n} 0}$ with the shock normal $\mathbf{n}$, and that a uniform upstream plasma flow $\mathbf{U}$ intersects the shock at an angle $\theta_{V \mathrm{n}}$. We assume that $\mathbf{B}_{0}, \mathbf{U}$ and $\mathbf{n}$ are coplanar, and lie within the xy-plane. The computations are first carried out in the de Hoffman-Teller frame of reference (deHoffmann and Teller, 1950), and then transformed into the plasma rest frame. Let $\mathbf{v}_{\mathbf{i}}^{\prime}$ and $\mathbf{v}^{\prime}$, respectively, denote the incident and reflected ion velocity in the de HoffmanTeller frame. Both velocities consist of components normal and tangential to the shock surface. The specular reflection from a stationary surface considers the reversal of the normal component while the tangential component remains unchanged (Gosling et al., 1982):

$v_{i n}^{\prime}=-v_{n}^{\prime}$

$v_{i t}^{\prime}=v_{t}^{\prime}$.

The Eqs. (1-2) are rotated in $\mathbf{B}_{0}$-related coordinates $\left(v_{\|}, v_{\perp 1}\right.$, $v_{\perp 2}$ ) and then transformed into the plasma frame of reference, where the incident ion velocities are indexed by the subscripts $i$ :

$$
\begin{aligned}
v_{i \|} & =-v_{\|} \cos 2 \theta_{B \mathrm{n} 0}-v_{\perp 2} \sin 2 \theta_{B \mathrm{n} 0}+2 V_{\mathrm{s}} \cos ^{2} \theta_{B \mathrm{n} 0} \\
v_{i \perp 1} & =v_{\perp 1} \\
v_{i \perp 2} & =-v_{\|} \sin 2 \theta_{B \mathrm{n} 0}-v_{\perp 2} \cos 2 \theta_{B \mathrm{n} 0}+V_{\mathrm{s}} \sin 2 \theta_{B \mathrm{n} 0},
\end{aligned}
$$

where $V_{\mathrm{s}}=-U \cos \theta_{V \mathrm{n}} / \cos \theta_{B \mathrm{n} 0}$.

If $f_{i}\left(\mathbf{v}_{i}\right)$ and $f(\mathbf{v})$ are, respectively, the initial and post encounter distribution functions, one has, according to Liouville's theorem,

$$
f(\mathbf{v})=f_{i}\left(\mathbf{v}_{i}\right)
$$

The thermal solar wind ions may be represented by an isotropic distribution in the form $f_{i}\left(\mathbf{v}_{i}\right) \sim \exp \left(-v_{i}^{2} / v_{\mathrm{th}}^{2}\right)$, where $v_{\text {th }}$ is related to the temperature of the distribution. 
According to Eq. (6), the post-reflection ion distribution is given by

$f\left(v_{\|}, v_{\perp 1}, v_{\perp 2}\right)=f_{i}\left(\mathbf{v}_{i}\left(v_{\|}, v_{\perp 1}, v_{\perp 2}\right)\right)$

which can be written as follows after using Eqs. (3-5)

$$
\begin{aligned}
& f\left(v_{\|}, v_{\perp 1}, v_{\perp 2}\right) \sim \exp \left[-\left\{\left(v_{\|}+2 V_{\mathrm{s}} \cos ^{2} \theta_{B \mathrm{n} 0}\right)^{2}+v_{\perp 1}^{2}\right.\right. \\
& \left.\left.\quad+\left(v_{\perp 2}+2 V_{\mathrm{s}} \cos \theta_{B \mathrm{n} 0} \sin \theta_{B \mathrm{n} 0}\right)^{2}\right\} / v_{\mathrm{th}}^{2}\right]
\end{aligned}
$$

The distribution function obtained corresponds to the initial distribution drifting with a velocity $\mathbf{v}_{\mathbf{0}}=-2 U \cos \theta_{V \mathrm{n}}\left[\cos \theta_{B \mathrm{n} 0}, 0, \sin \theta_{B \mathrm{n} 0}\right], \quad$ i.e. the center of the distribution is at a pitch-angle $\alpha_{0}=\theta_{B \mathrm{n} 0}$. Observationally, pitch-angle distributions and shock geometries are determined independently, and therefore can provide a test for the specular reflection hypothesis. The gyrating ions in Fig. 2 have pitch-angle distributions peaked at $\alpha_{0} \sim 30^{\circ}$, in agreement with the angle $\theta_{B \mathrm{n} 0}$ determined in Sect. 4. We note that the specular reflection mechanism as outlined here is a zero-order explanation for the production of gyrophase-bunched ions, and does not, for example, account for the gyrating ion temperatures that are greater than those for the solar wind source.

\section{Discussion}

One to six keV upstream ion flux modulations were first reported by Potter (1985), using observations from the onedimensional, very high time resolution Berkeley-Toulouse ion detector on board ISEE. In his study, Potter (1985) suggested that the ions should be in cyclotron resonance with the low-frequency waves accompaning them. Further studies showed that the flux modulation reported by Potter (1985) is associated with gyrophase-bunched ions (Fuselier et al., 1986c), suggesting that the ions could be trapped by the waves. The wave-trapping process causes all particle phase angles to be localized within a limited range of values (Berchem and Gendrin, 1985). When the magnetic field is perpendicular to a detector, such as for the BerkeleyToulouse instrument, that has a fixed orientation, trapped distributions will be observed to have high fluxes only during a limited range of the wave phases, and therefore, the particle count rate will be modulated.

Recently, Meziane et al. (2001) reported on observations by the 3-D ion analyzers on Wind/3DP of gyrophaserestricted particle distributions observed in association with flux modulations. According to their analysis, this event was consistent with a wave-trapping process, although other effects leading to the flux modulation could not be dismissed. In the next section we examine the possibility that the flux modulated ions may be due to changing conditions at the shock, which inhibit the ion escape.

\subsection{Cyclotron resonance}

Consistent with earlier investigations (Thomsen et al., 1985; Fuselier et al., 1986a; Mazelle et al., 2000, 2003; Meziane et al., 2001), the gyrating ion distributions discussed here are associated with highly transverse, weakly compressive lowfrequency waves propagating at small angles relative to the IMF. The angle $\theta_{k V}$ between the wave vector and the solar wind velocity has been computed by using the proton velocity averaged over the time interval studied. We point out that this angle is $\theta_{k V}=130 \pm 2^{\circ}$ (Table 1), which implies a propagation upstream in the solar wind frame. In order to investigate quantitatively the possibility that local cyclotron resonance between the waves and backstreaming ions produces the gyrating populations, we compare the wave periods $T_{\mathrm{obs}}$ with the periods $T_{\text {pred }}$ predicted for the spacecraft frame.

Consideration of the branches of the dispersion relation in the Brillouin plane helps to determine what kind of wave mode is a good candidate for cyclotron resonance (e.g. Brinca, 1991) The waves are quasi-monochromatic with large amplitudes, and are mainly transverse, which is consistent with the statistical study by Fuselier et al. (1986a). The variations of the ion density appear correlated with the magnetic field magnitude for the entire interval shown in Fig. 1, which is a property of the fast magnetosonic mode in the MHD limit. The properties of the associated waves could first lead to a search for wave excitation by an initial gyrophase-bunched ion (i.e. a highly nongyrotropic) distribution. Over a wide range of values for the mean pitch-angle of the gyrating ions, the instability which gives rise to the largest growth rate is the ion-ion right-hand helical beam instability (e.g. Gary, 1991, and references therein). This type of instability has also been reported around comets, generated by the newborn heavy ions near their gyrofrequency (e.g. Brinca, 1991; Tsurutani, 1991; Cao et al., 1998, and references therein), as well as at the proton gyrofrequency (Mazelle and Neubauer, 1993). This mode is basically the same for a field-aligned beam (Gary et al., 1981; Brinca, 1991). The wave mode is necessarily right-handed and costreaming with the ions along the ambient magnetic field, i.e. away from the shock, so that it can resonate with a backstreaming ion population ( $\mathbf{k} \cdot \mathbf{V}_{\|}$must be positive). In the MHD limit, this mode is the magnetosonic mode, and its phase velocity is of the order of the local Alfvèn speed, i.e. much smaller than the parallel ion speed $V_{\|}$, which is always larger than $V_{\mathrm{sw}}$. Therefore, the ion beam overtakes the waves so that there is an anomalous Doppler shift in its reference frame; the ions sense left-handed waves. Considering the small spacecraft velocity relative to the Earth, the proton right-hand mode waves are also observed in the spacecraft frame as left-handed waves. Moreover, for the MHD limit of this type of low-frequency (magnetosonic) wave, the group velocity is parallel to the magnetic field with the same sense as $\mathbf{k}_{\|}$; therefore, the wave energy (the Poynting flux) is driven away from the Earth's environment in the solar wind frame, contrary to solar wind-originated waves. This is fully consistent with the observed value of $\theta_{k V}$.

The cyclotron resonance condition with the right-hand mode is written:

$\omega-k_{\|} V_{\|}+\Omega_{p}=0$, 


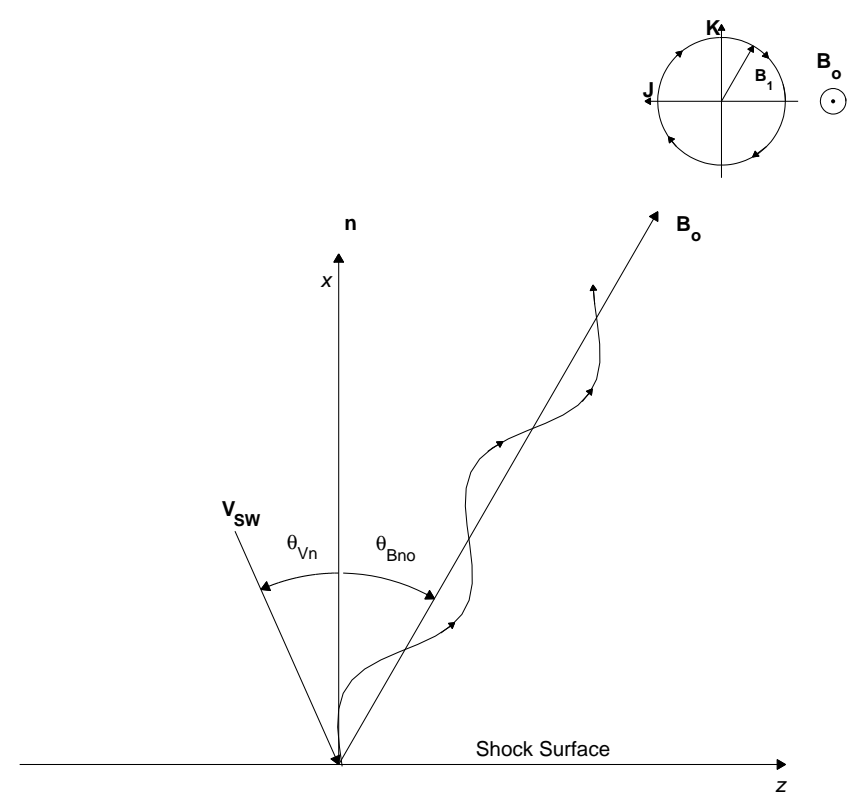

Fig. 4. A simple sketch showing the transverse wave upstream propagation from the shock and along the ambient magenetic field.

where $\omega$ is the wave frequency in the solar wind frame, $\Omega_{p}$ is the proton gyrofrequency, $k_{\|}$is the component of the wave vector parallel to the background magnetic field, and $V_{\|}$is the parallel component of the resonant ion velocity (solar wind frame). By first assuming $\omega \ll \Omega_{p}$, we can make the approximation

$k_{\|} \approx \Omega_{p} / V_{\|}$.

In the spacecraft frame, these waves would have a Dopplershifted frequency of

$\omega^{\prime}=\omega+\mathbf{k} \cdot \mathbf{V}_{\mathrm{sw}} \approx k_{\|} \mathbf{V}_{\mathrm{sw}} \cos \theta_{k V} / \cos \theta_{k B}$.

We have computed the predicted wave periods $T_{\text {pred }} \equiv 2 \pi / \omega^{\prime}$ according to Eqs. (10) and (11) and now compare it with the observed period $T_{\text {obs }}$.

Consider first the ion distributions. For 15:17:0015:19:44 UT, the gyrating protons have parallel velocities which are typically of the order of $V_{\|}=600 \pm 100 \mathrm{~km} \mathrm{~s}^{-1}$. This gives $T_{\text {pred }}=24 \mathrm{~s}$, which is very different from the observed period $T_{\mathrm{obs}}=47 \mathrm{~s}$.

We conclude that these gyrating distributions are not in cyclotron resonance with the observed ULF waves, and this rules out a coherent wave trapping mechanism to account for them. This is in contrast to other Cluster observations at similar distances from the bow shock (Mazelle et al., 2003). An additional argument against the local production of gyrating ions through nonlinear beam disruption by wave excition is that this should produce gyrophase-bunched distributions with a specific pitch-angle $\alpha_{0}$, which depends upon the wave amplitude (Mazelle et al., 2000). For $\alpha_{0}$ computed in the frame moving along $\mathbf{B}_{0}$ at the phase velocity $V_{\phi}$ of the wave, the theoretical value is $\alpha_{\text {theory }}=53^{\circ}$ (Mazelle et al., 2003), where we have used the mean value $\left|\delta B_{\perp}\right| / B_{0}=0.48$ from the observations. In contrast, the observed values are $\alpha_{\exp } \approx 30^{\circ}$.

We make the same test of period matching for the field-aligned beam observed at 15:16:48-56 UT for which $V_{\|}=1060 \pm 100 \mathrm{~km} \mathrm{~s}^{-1}$, which gives $T_{\text {pred }}=46.5 \mathrm{~s}$ while $T_{\mathrm{obs}}=46.6 \pm 2.0 \mathrm{~s}$ and leads to a very good agreement.

Applying Eq. 11 and assuming $\omega \ll \omega^{\prime}$ leads to a first estimate of the parallel wavelength $\lambda_{\| 1} \sim 12100 \mathrm{~km}$. Using Eq. (10), which assumes $\omega \ll \Omega_{p}$, leads to a second estimate $\lambda_{\| 2} \sim 11660 \mathrm{~km}$. Moreover, if one solves the linear system made by Eqs. (9) and (11) without any approximation, assuming exact local cyclotron resonance, this leads to a plasma frame wave frequency $\omega / \Omega_{p}=0.02$ and a parallel wavelength $\lambda_{\| 3}=11526 \mathrm{~km}$, i.e. wave properties that are fully consistent with the hypotheses made. This strongly supports the possibility that such a beam distribution could have generated the observed waves from the resonant right-hand ion/ion beam instability. The waves propagating upstream in the solar wind frame with a phase speed much smaller than $V_{\text {sw }}$ are simply blown back by the solar wind and registered locally with the local backstreaming distributions leaking upstream with a much larger parallel velocity $\left(V_{\|} \sim 10 V_{\mathrm{A}}\right.$, typically).

\subsection{Local bow shock geometry}

Wave modes as described above may be excited in an ionion cylotron instability due to field-aligned beams that propagate upstream having appropriate properties. Because they are convected back to the shock, and due to their substantial wave amplitude, they can modulate the instantaneous local shock geometry $\left(\theta_{B \mathrm{n}}\right)$ in phase with the wave. To take into account this effect on the shock geometry, we consider the geometrical model proposed by Fuselier et al. (1986b). A monochromatic low-frequency MHD wave intrinsically right-handed in the solar wind frame propagates upstream roughly along the ambient magnetic field $\mathbf{B}_{0}$. The wave is assumed to be purely transverse with a wave vector $\mathbf{k} \| \mathbf{B}_{0}$ and a phase velocity $V_{\varphi}=\omega / k$, where $\omega$ is the wave frequency in the plasma rest frame of reference. In the spacecraft frame of reference the wave is left-handed due to Doppler-shifting, and the measured wave frequency $\omega_{\mathrm{sc}}$ satisfies $\omega=\omega_{\mathrm{sc}}-\mathbf{k} \cdot \mathbf{V}_{\mathrm{sw}}$. The simple sketch shown in Fig. 4 illustrates the transverse upstream wave propagation from the shock surface.

In the coordinate system associated with the minimum variance frame, the components of the ULF wave written in the spacecraft frame are

$$
\begin{aligned}
& B_{I}=0 \\
& B_{J}=\eta B_{0} \cos \left(\mathbf{k} \cdot \mathbf{r}-\omega_{\mathrm{sct}}+\phi_{0}\right) \\
& B_{K}=-\eta B_{0} \sin \left(\mathbf{k} . \mathbf{r}-\omega_{\mathrm{sct}}+\phi_{0}\right),
\end{aligned}
$$

where $\eta$ is related to the wave amplitude $\eta=|\delta \mathbf{B}| / B$. Here we neglect the parallel component $B_{I}$; taking into account this component leads to no significant changes in the results. Using the measured magnetic field and particle velocities, we 
estimate the instantaneous magnetic field at the shock, given the distance from it. We assume here that the wave train remains coherent between the observation point and the shock surface. We justify this assumption by noting that according to the three bow shock models, briefly described above, the Cluster SC 1 is located at less than a wavelength from the bow shock. At each instant corresponding to the times when distributions are measured, we deduce the wave phase at the shock. The components at the shock of the magnetic field associated with the waves written in the minimum variance frame are then:

$$
\begin{aligned}
& B_{I}=0 \\
& B_{J}=\eta B_{0} \cos \left(\mathbf{k} \cdot \mathbf{r}_{\mathbf{S}}-\omega_{\mathrm{sc}} D_{S} / v_{\|}+\phi_{0}\right) \\
& B_{K}=-\eta B 0 \sin \left(\mathbf{k} \cdot \mathbf{r}_{\mathbf{S}}-\omega_{\mathrm{sc}} D_{S} / v_{\|}+\phi_{0}\right),
\end{aligned}
$$

where $D_{S}$ is the distance computed along $\mathbf{B}_{0}$ from the shock to the observation point, $\mathbf{r}_{\mathrm{S}}$ is the position of the point $S$ where the IMF intersects the shock, and $V_{\|}$is the measured particle parallel velocity, which is close to the particle velocity component along the minimum variance axis.

The vector $\mathbf{r}_{\mathrm{S}}$ is obtained from the values given in Table 2, after rotation from the aberrated-GSE frame into the minimum variance frame. Finally, the wave field is added to $\mathbf{B}_{0}$ to obtain the total magnetic field at the shock at a given time, and hence we deduce an estimate of $\theta_{B \mathrm{n}}$. Figure 5 shows estimates of $\theta_{B \mathrm{n}}$ variations at the shock during the time interval where the ULF waves are present.

The Cairns et al. (1995) bow shock model has been used for the computation. The computations based on Slavin and Holzer (1981); Farris et al. (1991) led to similar results. The horizontal dashed line corresponds to the critical value of $\theta_{B \mathrm{n}}^{c}=39.9^{\circ}$. On the same figure, we report the particle count rate data associated with the $1.3 \mathrm{keV}$ channel from CIS/HIA. Figure 5 clearly shows that the obtained $\theta_{B \mathrm{n}}$ values are anti-correlated with the particle count rates. The shock geometry periodically switches from quasi-parallel $\left(\theta_{B \mathrm{n}}<45^{\circ}\right)$ to quasi-perpendicular $\left(\theta_{B \mathrm{n}}>45^{\circ}\right)$ geometries; the highest count rates are observed for quasi-parallel geometries. Above the critical theoretical value of $\theta_{B \mathrm{n}}^{c}=39.9^{\circ}$, the count rates are significantly lower at maximum $\theta_{B \mathrm{n}}$. These results strongly indicate that the particle emission efficiency is dependent upon the instantaneous shock geometry. It is clear that the region defined by $\theta_{B \mathrm{n}} \leq 45^{\circ}$ is associated with higher emission efficiencies. Because the average particle velocities are consistent with the specular reflection mechanism and with the $\theta_{B \mathrm{n}}$ temporal variations reported in Fig. 5, we interprete the observed count rate oscillations as a result from varying escape rates at the shock due to changing local shock geometry.

\section{Conclusions}

We have examined the particle count rate modulation observed upstream of the bow shock by the Cluster-CIS experiment. Previous studies on particle modulation observed

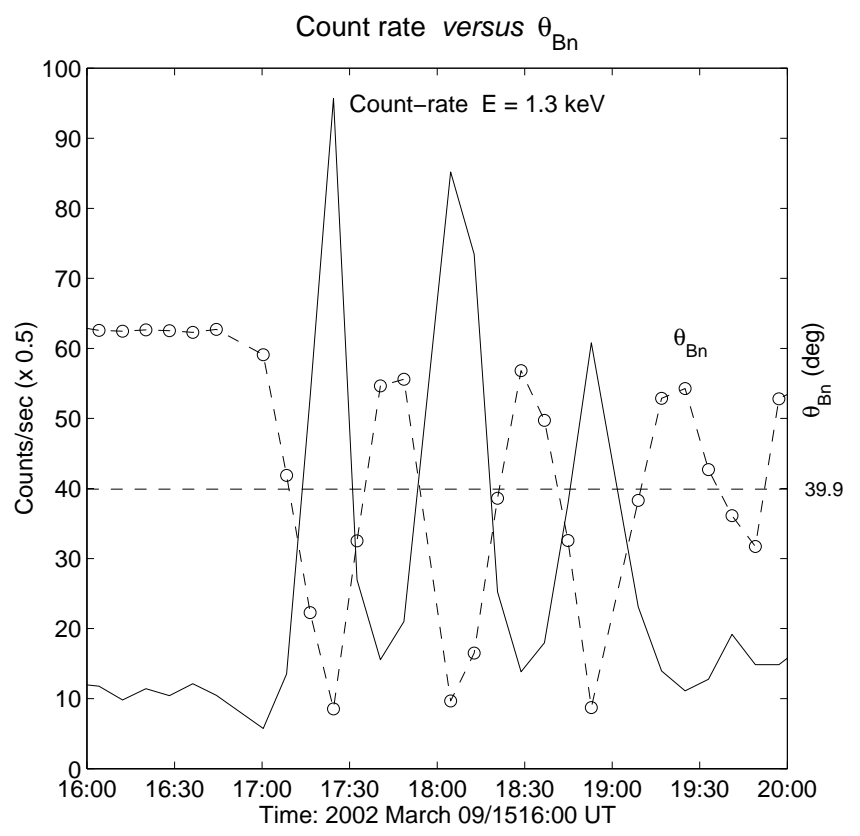

Fig. 5. The changes in local shock $\theta_{B \mathrm{n}}$ angle (dashed line-open circles) and the particle count rates during the interval when the ULF waves are present. The dashed horizontal line corresponds to $\theta_{B \mathrm{n}}^{c}=39.9^{\circ}$. For clarity, the particle count rate values have been scaled by a factor 0.5 .

upstream of the bow shock suggested possibilities of particle wave-trapping resulting from a very coherent interaction between waves and particles (Fuselier et al., 1986c; Meziane et al., 2001; Mazelle et al., 2003). We have conducted detailed analyses on both protons and the associated waves that are responsible for the count rate modulations.

The particle distributions have pitch-angles peaked at $\sim 30^{\circ}$, which is inconsistent with wave trapping theory. Instead, the parallel and the perpendicular velocity components are consistent with the specular reflection hypothesis, placing the production source at the shock. We have found no evidence that the gyrating ions are trapped by the nearly monochromatic ULF waves. In fact, these waves do not resonate with the particles that they accompany, but rather do so with the field-aligned beams further upstream. This strongly suggests that the source of these waves is the dispruption of the earlier field-aligned beams. The wave field is convected downstream towards the shock and therefore affects the local conditions at the shock. We have assumed that the observed wave properties are nearly conserved over the short distance $\left(D_{S}<\lambda_{\|}\right)$that the wave train is convected, and have modelled their propagation to the shock. An underlying assumption, consistent with our wave analysis, is that the interaction between the waves and particles over this short distance is unimportant. We then estimated the local instantaneous $\theta_{B \mathrm{n}}$ angle, and have found that high count rates were associated with $\theta_{B \mathrm{n}}$ less than $\sim 40^{\circ}$, consistent with escaping specularly reflected protons. For $\theta_{B \mathrm{n}} \geq 40^{\circ}$, specularly reflected ions are expected to be unable to escape upstream, since their orbits should reencounter the shock. This accounts for the intermittent character of the particle count rate. 
Acknowledgements. K. Meziane and C. Mazelle thank ISSI for support. K. Meziane thanks CNRS for supporting his visit to CESR. Work at UNB was funded by grants from the Canadian Natural Science and Engineering Research Council. K. Meziane thanks E. Penou for his assitance.

Topical Editor T. Pulkkinen thanks two referees for their help in evaluating this paper.

\section{References}

Balogh, A., Carr, C. M., Acũna, M. H., Dunlop, M. W., Beek, T. J., Brown, P., Fornacon, K.-H., Georgescu, E., Glassmeier, K.-H., Harris, J., Musmann, G., Oddy, T., and Schwingenschuh, K.: The cluster magnetic field investigation: overview of in-flight performance and initial results, Ann. Geophys., 19, 1207-1217, 2001.

Berchem, J. and Gendrin, R.: Nonresonant interaction of heavy ions with electromagnetic ion cyclotron waves, J. Geophys. Res., 90, 10 945-10 960, 1985.

Bonifazi, C. and Moreno, G.: Reflected and diffuse ions backstreaming from the Earth's bow shock, 1, basic properties, J. Geophys. Res., 86, 4381-4396, 1981a.

Brinca, A. L.: Cometary linear instabilities: from profusion to prospective, in Cometary Plasma Processes, edited by Johnstone, A. D., Geophysical Monograph 61, pp. 211-221, American Geophysical Union, 1991.

Cairns, I. H., Fairfield, D. H., Anderson, R. R., Carlton, V. E. H., Paularena, K. I., and Lazarus, A. J.: Unusual locations of Earth's bow shock on september 24-25, 1987: Mach number effects, J. Geophys. Res., 100, 47-62, 1995.

Cao, J., Mazelle, C., Belmont, G., and Rème, H.: Oblique ring instability driven by nongyrotropic ions: application to observations at comet grigg-skjellerup, J. Geophys. Res., 103, 20552067, 1998.

deHoffmann, F. and Teller, E.: Magneto-hydrodynamic shocks, Physical Review, 80, 692-703, 1950.

Eastwood, J., Balogh, A., Dunlop, M. W., Horbury, T. S., and Dandouras, I.: Cluster observations of fast magnetosonic waves in the terrestrial foreshock, Geophys. Res. Lett., 29, 2046, doi:10.1029/2002GL015 582, 2002.

Eastwood, J., Balogh, A., Lucek, E., Mazelle, C., and Dandouras, I.: On the existence of Alfv'en waves in the terrestrial foreshock, Ann. Geophys., 21, 1457-1465, 2003.

Fairfield, D. H.: Average and unusual locations of the Earth's magnetopause and bow shock, J. Geophys. Res., 76, 6700-6716, 1971.

Farris, M. H., Petrinec, S. M., and Russell, C. T.: The thickness of the magnetosheath: constraints on the polytropic index, Geophys. Res. Lett., 18, 1821-1824, 1991.

Formisano, V.: Orientation and shape of the Earth's bow shock in three dimension, Planet. Space Sci., 27, 1151-1161, 1979.

Fuselier, S. A.: Suprathermal ions upstream and downstream from the Earth's bow shock, in Solar Wind Sources of Magnetospheric Ultra-Low Frequency Waves, edited by Engebretson, M., Takahashi, M., and Scholer, M., Geophysical Monograph 81, pp. 91114, American Geophysical Union, 1994.

Fuselier, S. A., Thomsen, M. F., Gosling, J. T., Bame, S. J., and Russell, C. T.: Gyrating and intermediate ion distributions upstream from the Earth's bow shock, J. Geophys. Res., 91, 91-99, 1986a.
Fuselier, S. A., Gosling, J. T., and Thomsen, M. F.: The motion of ions specularly reflected off a quasi-parallel shock in the presence of large-amplitude, monochromatic mhd waves, J. Geophys. Res., 91, 4163-4170, 1986b.

Fuselier, S. A., Thomsen, M. F., Gary, S. P., Bame, S. J., Russell, C. T., and Parks, G. K.: The phase relationship between gyrophase-bunched ions and mhd-like waves, Geophys. Res. Lett., 13, 60-63, 1986c.

Gary, S. P.: Electromagnetic ion/ion instabilities and their consequences in space plasmas: A review, Space Science Reviews, 56, 373-415, 1991.

Gary, S. P., Gosling, J. T., and Forslund, D. W.: The electromagnetic ion beam instability upstream of the Earth's bow shock, J. Geophys. Res., 86, 6691-6696, 1981.

Gosling, J. T. and Robson, A. E.: Ion reflection, gyration, and dissipation at supercritical shocks, in Collisionless shocks in the heliosphere: reviews of current research, edited by Tsurutani, B. T. and Stone G. S., Geophysical Monograph 35, pp. 141-152, American Geophysical Union, 1985.

Gosling, J. T., Thomsen, M. F., Bame, S. J., Feldman, W. C., Paschmann, G., and Sckopke, N.: Evidence for speculary reflected ions upstream from the quasi-parallel bow shock, Geophys. Res. Lett., 87, 1333-1336, 1982.

Greenstadt, E. W. and Mellott, M. M.: Variable field-to-normal shock-foreshock boundary observed by isee-1 and -2, Geophys. Res. Lett., 12, 129-132, 1985.

Gurgiolo, C., Parks, G. K., and Mauk, B. H.: Upstream gyrophase bunched ions: A mechanism for creation at bow shock and the growth of velocity space structure through gyrophase mixing, J. Geophys. Res., 88, 9093-9100, 1983.

Hoshino, M. and Terasawa, T.: Numerical study of the upstream waves excitation mechanism, 1, nonlinear phase bunching of beam ions, J. Geophys. Res., 90, 57-64, 1985.

Mailing, D. H.: Coordinate Systems and Map Projections, Pergamon, Tarrytown, N. Y., 2nd edn., 1992.

Mazelle, C. and Neubauer, F. M.: Discrete wave packets at the proton cyclotron frequency at comet p/halley, Geophys. Res. Lett., 20, 153-156, 1993.

Mazelle, C., LeQuéau, D., and Meziane, K.: Nonlinear waveparticle intereraction upstream from the Earth's bow shock, Nonlinear Processes Geophys., 7, 185-190, 2000.

Mazelle, C., Meziane, K., LeQuéau, D., Wilber, M., Eastwood, J. P., Rème, H., Sauvaud, J.-A., Bosqued, J.-M., Dandouras, I., McCarthy, M., Kistler, L. M., Klecker, B., Korth, A., BavassanoCattaneo, M. B., Lundin, R., and Balogh, A.: Production of gyrating ions from nonlinear wave-particle interaction upstream from the Earth's bow shock: a case study from Cluster-CIS, Planet. Space Sci., pp. 785-795, 2003.

Merka, J., Szabo, A., Narock, T. W., King, J. H., Paularena, K. I., and Richardson, J. D.: A comparison of IMP 8 observed bow shock positions with model predictions, J. Geophys. Res., 108, 1077, doi:10.1029/2002JA009384, 2003.

Meziane, K., Mazelle, C., Lin, R. P., LeQuéau, D., Larson, D. E., Parks, G. K., and Lepping, R. P.: Three-dimensional observations of gyrating ion distributions far upstream from the Earth's bow shock and their associated with low-frequency waves, J. Geophys. Res., 106, 5731-5742, 2001.

Peredo, M., Slavin, J. A., Mazur, E., and Curtis, S. A.: Threedimensional position and shape of the bow shock and their variation with alfvénic, sonic and magnetosonic mach numbers and interplanetary magnetic field orientation, J. Geophys. Res., 100, 7907-7916, 1995. 
Potter, D. W.: High time resolution characteristics of intermediate ion distributions upstream of the Earth's bow shock, J. Geophys. Res., 90, 261-265, 1985.

Rème, H., Aoustin, C., Bosqued, J. M., Dandouras, I. et al.: First multispacecraft ion measurements in and near the Earth's magnetosphere with identical Cluster ion spectrometry (CIS) experiment, Ann. Geophys., 19, 1303-1354, 2001.

Schwartz, S. J., Thomsen, M. F., and Gosling, J. T.: Ions upstream of the Earth's bow shock: a theoretical comparison of alternative source populations, J. Geophys. Res., 88, 2039-2047, 1983.

Slavin, J. H. and Holzer, R. E.: Solar wind flow about terrestrial planets: 1. modeling bow shock position and shape, J. Geophys. Res., 86, 11401-11418, 1981.
Sonnerup, B. U. and Scheible, M.: Analysis Methods for MultiSpacecraft Data, chap. Minimum and Maximum Variance Analysis, pp. 185-220, ISSI Scientific Report, ESA Publications Division, Keplerlaan 1, 2200 AG Noordwijk, The Netherlands, 1998.

Thomsen, M. F., Gosling, J. T., Bame, S. J., and Russell, C. T.: Gyrating ions and large-amplitude monochromatic MHD waves upstream of the Earth's bow shock, J. Geophys. Res., 90, 267273, 1985.

Tsurutani, B. T.: Comets: A laboratory for plasma waves and instabilities, cometary plasma processes, in Cometary Plasma Processes, edited by Johnstone, A. D., Geophysical Monograph, pp. 189-209, American Geophysical Union, 2000 Florida Avenue N.W., Washington, DC 20009, 1991. 